\title{
Determinação de resveratrol e características químicas em sucos de uvas produzidas em sistemas orgânico e convencional
}

\author{
Andréia Andrade de Freitas ${ }^{1}$, Alessandra Maria Detoni², Edmar Clemente ${ }^{3}$, Cláudio Celestino de Oliveira ${ }^{3}$
}

\section{RESUMO}

O presente estudo avaliou os teores de trans-resveratrol e algumas características químicas: acidez total titulável (ATT), sólidos solúveis totais (SST) e relação SST/ATT, em amostras de sucos de uvas da variedade Concorde e Rúbea, sobre o porta-enxerto Campinas IAC 766, produzidas em sistemas orgânico e convencional. O suco foi obtido pelo método de cocção de uvas sadias e maduras. Os resultados encontrados para os sucos de uvas das variedades Concorde e Rúbea, sobre o porta-enxerto Campinas IAC 766, orgânico e convencional, apresentaram concentração de trans-resveratrol de 25,9; 27,2; 32,5 e 32,9 mg L-1 , respectivamente. No suco das variedades Concorde e Rúbea de produção orgânica foram obtidos os valores de 0,77 e 0,91 g de ácido tartárico $100 \mathrm{~mL}^{-1}$. Para as mesmas variedades produzidas em sistema convencional 0,94 e 0,75 g de ácido tartárico $100 \mathrm{~mL}^{-1}$. Os valores observados para SST e SST/ ATT para as variedades de sistemas orgânico e convencional foram 10,95; 11,73; 14,23; e 13,23 ${ }^{\circ}$ Brix e 14,22; 12,64; 15,18; e 17,64, respectivamente. Das avaliações realizadas com relação ao trans-resveratrol pode-se concluir que as variedades, independentemente do sistema de cultivo, são fontes de resveratrol e que os sucos podem ser consumidos como alimento funcional.

Palavras-chave: Trans-resveratrol, suco de uvas, cultivo orgânico e convencional

\section{ABSTRACT}

\section{Determination of resveratrol and chemical characteristics in juice from grapes produced under organic and conventional systems}

The present study was carried out to evaluate the contents of trans-resveratrol and the chemical characteristics total tritable acidity (TTA), total soluble solids (TSS) and TSS/TTA, in samples of juice from grapes produced under organic and conventional systems. Juice was obtained by the method of cooking healthy and ripe grapes. Juice made from grapes of the varieties Concord and Ruby (on IAC 766 rootstock) cultivated under organic and conventional systems, showed trans-resveratrol concentrations of 25.9; 27.2; 32.5 and $32.9 \mathrm{mg} \mathrm{L}^{-1}$, respectively. Juice from Concord and Ruby in the organic production produced 0.77 and $0.91 \mathrm{~g}$ tartaric acid/100 $\mathrm{mL}^{-1}$. The same varieties, in the conventional system, produced 0.94 and $0.75 \mathrm{~g}$ tartaric acid/100 $\mathrm{mL}^{-1}$. SST and SST/ATT for the varieties under organic and conventional systems were $10.95,11.73,14.23$ and $13.23^{\circ}$ Brix and 14.22, 12.64, 15.18 and $17.64{ }^{\circ}$ Brix, respectively. The evaluations showed that the varieties, independent of cropping system, are sources of trans-resveratrol and the produced juice can be consumed as a functional food.

Key words: Trans-resveratrol, grape juice, organic and conventional cultivation

\footnotetext{
Recebido para publicação em julho de 2007 e aprovado em novembro de 2009

${ }^{1}$ Tecnóloga em Alimentos, Mestre. Universidade Estadual de Maringá, Centro de Ciências Exatas, Departamento de Química. Av. Colombo, 5790, Zona 7, 87020-900, Maringa, PR, Brasil. andreia.afreitas@gmail.com

${ }^{2}$ Engenheira Agrônoma, doutora. Universidade Estadual de Maringá, Centro de Ciências Exatas, Departamento de Química. Av. Colombo, 5790, Zona 7, 87020-900, Maringa, PR, Brasil. alessandradetoni@yahoo.com.br

${ }^{3}$ Bacharel em Química, Doutor. Universidade Estadual de Maringá, Centro de Ciências Exatas, Departamento de Química. Av. Colombo, 5790, Zona 7, 87020-900, Maringa, PR, Brasil. eclemente@uem.br Autor para correspondência.
} 


\section{INTRODUÇÃO}

A preocupação com uma alimentação saudável ocasiona a busca por alimentos que ofereçam propriedades que vão além de nutrir, mas também proteger o organismo de enfermidades. Nesse contexto algumas pesquisas têm demonstrado o importante papel do resveratrol, molécula presente em vinhos e sucos de uvas na prevenção de doenças, principalmente aquelas ligadas ao sistema cardiovascular.

No organismo humano o resveratrol traz benefícios ligados ao coração, como a hipoagregação de plaquetas e a inibição da peroxidação de lipoproteínas de baixa densidade (Kolouchov-Hanzlikov et al., 2004; Possier, 2005). Outros importantes benefícios também estão sendo estudados, comprovando o seu poder anticancerígeno pelo aumento da apoptose e detenção da multiplicação celular, inibindo a proliferação de células epiteliais malígnas da mama, células do câncer de próstata e tumor do cólon (Possier, 2005; Sauter, 2005).

Sintetizada por plantas superiores, a molécula de resveratrol (trans - 3,5,4-trihydroxystilbene) é um polifenol antioxidante (Wang, 2002, Nikfardjam et al., 2006). Esse elemento químico encontrado na videira é acumulado nos tecidos das folhas, atuando como agente protetor da planta contra os raios ultravioletas, as injúrias mecânicas e o ataque fúngico causado, principalmente, por Botrytis cinerea e Plasmospora viticola. O resveratrol atua na inibição do progresso da infecção, o que o inclui numa classe de antibióticos naturais chamado fitoalexina (KolouchovHanzlikov et al., 2004).

Na vitivinicultura orgânica, o uso de alguns produtos químicos é proibido, de acordo com a Instrução Normativa n. 007 do Ministério da Agricultura (Brasil, 1974), que dispõe sobre as normas para a produção de orgânicos, é vedado o uso de agrotóxicos sintéticos no controle de pragas e doenças, as quais são comumente controladas, ou apenas amenizadas, com caldas e preparados naturais. Dessa forma, as plantas cultivadas no sistema orgânico de produção necessitam dispor de seus mecanismos internos de defesa. O mecanismo natural de defesa das plantas é obtido por meio do seu metabolismo secundário, com a produção de determinados compostos químicos como por exemplo, o resveratrol.

De modo geral, concentrações significativas de resveratrol são encontradas nos vinhos e sucos de uvas brasileiros, porém essas concentrações variam de acordo com a origem e o tipo da uva, o processo de vinificação ou extração do suco e a infecção fúngica ocorrente na videira. Levando-se em consideração o sistema orgânico de produção, espera-se que a videira conduzida neste esquema produza maior quantidade desse composto fenólico.
Diante disto, o objetivo deste estudo foi avaliar a quantidade de resveratrol e algumas características químicas de sucos de uvas obtidos de diferentes cultivares, produzidos nos sistemas orgânico e convencional.

\section{MATERIAL E MÉTODOS}

As uvas utilizadas no experimento foram colhidas na fazenda experimental da Universidade Estadual de Maringá (UEM), Maringá, PR. As variedades usadas foram Concorde e Rúbea, sobre o porta-enxerto Campinas IAC 766, produzidas em sistemas orgânico e convencional.

A colheita foi realizada quando as uvas apresentavam, em média, 14 B Brix. Depois de colhidos, os cachos foram levados ao Laboratório de Bioquímica de Alimentos da UEM, onde foram sanitizados e submetidos à obtenção do suco por processo de cocção. Os sucos foram engarrafados a quente, em uma temperatura suficiente para garantir a estabilidade biológica e a conservação sem aditivos químicos (Rizzon, 1998).

Após a extração do suco de uva das diferentes variedades, as amostras foram homogeneizadas e realizadas as determinações de sólidos solúveis totais (SST) e acidez total titulável (ATT) e calculado o ratio (SST/ATT), segundo o método descrito pelo Instituto de Tecnologia de Alimentos (Ital, 1990).

A determinação do trans-resveratrol foi realizada segundo metodologia reportada por Souto et al. (2001). Os padrões foram preparados e filtrados em membranas de porosidade $0,22 \mathrm{~m} \mu$.

Para a obtenção dos cromatogramas foi utilizado cromatógrafo líquido Varian ProStar modular contendo: bomba de pistão reciprocante, modelo 240 série 00752, injetor automático Varian (Auto Sample ProStar 410) com alça de amostragem de $20 \mu \mathrm{L}$, coluna cromatográfica RP18 e detector espectrofotométrico modelo ProStar 350 com arranjo de fotodiodo e programa de aquisição de dados Polyview. Na fase móvel foi utilizada acetonitrila 75:25 em $1,5 \mathrm{~mL} \mathrm{~min}^{-1}$. Nessas condições o tempo de retenção do tresveratrol foi de 9,6 $\mathrm{min}$.

\section{RESULTADOS E DISCUSSÃO}

O rendimento do suco foi de $60 \%$. Na avaliação das características químicas dos sucos de uvas (Tabela 1) foi verificado, de modo geral, baixos teores de sólidos solúveis totais (SST), variando de 10,95 a 14,23 ${ }^{\circ}$ Brix. Estes valores podem ser justificados pelo método de obtenção do suco, o qual foi obtido pelo seu processo de cocção. Nesse processo pode ocorrer diluição do material devido ao vapor de água utilizado no aquecimento para extração do suco. Esse método de cocção é comumente utilizado em pequenas propriedades produtoras de suco de uva. 
Tabela 1. Características químicas de sucos de uva orgânicos e convencionais, comparados aos limites da legislação brasileira

\begin{tabular}{|c|c|c|c|c|}
\hline \multirow{2}{*}{ Sistema cultivo } & \multirow{2}{*}{ Convencional } & \multirow{2}{*}{ Orgânico } & \multicolumn{2}{|c|}{ Limites da legislação } \\
\hline & & & Mín. & Máx. \\
\hline \multicolumn{5}{|c|}{ SST $^{1}$} \\
\hline Concorde 766 & 10,95 & 14,23 & \multirow{2}{*}{14,0} & \multirow{2}{*}{ - } \\
\hline Rúbea 766 & 11,73 & 13,23 & & \\
\hline \multicolumn{5}{|c|}{ ATT $^{2}$} \\
\hline Concorde 766 & 0,77 & 0,94 & \multirow{2}{*}{-} & \multirow{2}{*}{0,90} \\
\hline Rúbea 766 & 0,91 & 0,75 & & \\
\hline \multicolumn{5}{|c|}{ SST / ATT } \\
\hline Concorde 766 & 14,22 & 15,18 & \multirow[b]{2}{*}{15,0} & \multirow[b]{2}{*}{45,0} \\
\hline Rúbea 766 & 12,64 & 17,64 & & \\
\hline
\end{tabular}

${ }^{1}$ SST - Sólidos solúveis totais expressos em ${ }^{\circ}$ Brix. e ${ }^{2} \mathrm{ATT}$ - Acidez total titulável expressa em g de ácido tartárico $100 \mathrm{~mL}^{-1}$ de suco.

De acordo com a legislação brasileira para sucos de uva, o valor de SST deve ser no mínimo 14 Brix (Brasil, 1974). Dessa forma, apenas o suco obtido da variedade Concorde no sistema convencional (Tabela 1) está de acordo com o exigido nos padrões de qualidade. Porém esse cultivar apresentou teor médio de acidez total titulável (ATT) de 0,94 g de ácido tartárico em 100 mL de suco, superior ao permitido pelo padrão nacional, que admite no máximo 0,90 g em $100 \mathrm{~mL}^{-1}$ de suco. Mesmo assim a relação SST/ATT $(15,18)$ ficou nos limites da legislação, que admite uma variação de 15,0 a 45,0 nessa relação.

De acordo com Pezzi e Fenocchio (1976), a relação entre SST/ATT representa o equilíbrio entre o gosto doce e o ácido do suco de uva, portanto um indicativo da qualidade. Neste experimento as variedades Concorde e Rúbea, no sistema convencional, apresentam relação SST /ATT de 15,18 e 17,64, respectivamente, estando de acordo com os padrões estabelecidos na legislação.

Em relação aos teores de SST, as variedades orgânicas estão semelhantes aos descritos por Rizzón \& Link (2006), (12,2-13, $1^{\circ}$ Brix), os quais obteveram os sucos pelo mesmo processo de extração, devido ao efeito da diluição do vapor d’água em decorrência do tipo de equipamento utilizado.

Em relação ao trans-resveratrol, a análise foi conduzida em HPLC, resultando em boa resolução e curto tempo de análise, como pode ser observado no cromatograma da Figura 1.

Os resultados de trans-resveratrol obtidos para os sucos analisados encontram-se na Tabela 2 e demonstram que não ocorreram grandes diferenças entre os teores de trans-resveratrol para as duas variedades (Concord e Rúbea) nos diferentes sistemas de cultivo. Foi observado, em média, 25,9 a 32,9 mg de transresveratrol por litro de suco. Embora não tenham ocorrido grandes diferenças entre as variedades e os sistemas de cultivo, os dados obtidos são superiores aos descritos na literatura. Yasui et al. (1997) e Romero-Pérez et al. (1999), ao avaliarem os níveis de resveratrol em vários sucos de uvas comerciais, obtiveram teor máximo de resveratrol de 14,47 $\mathrm{mg} \mathrm{L}^{-1}$ e 0,44 $\mathrm{mg} \mathrm{L}^{-1}$, respectivamente. Lamuela-Raventós et al. (1995) e Romero-Pérez et al. (1996), com vinhos tintos espanhóis, obtiveram 5,65 mg $\mathrm{L}^{-1}$ de resveratrol. Porém em vinhos, de acordo com Rodrigues-Delgado et al. (2002), a quantidade de resveratrol é inferior à encontrada nos sucos de uvas.

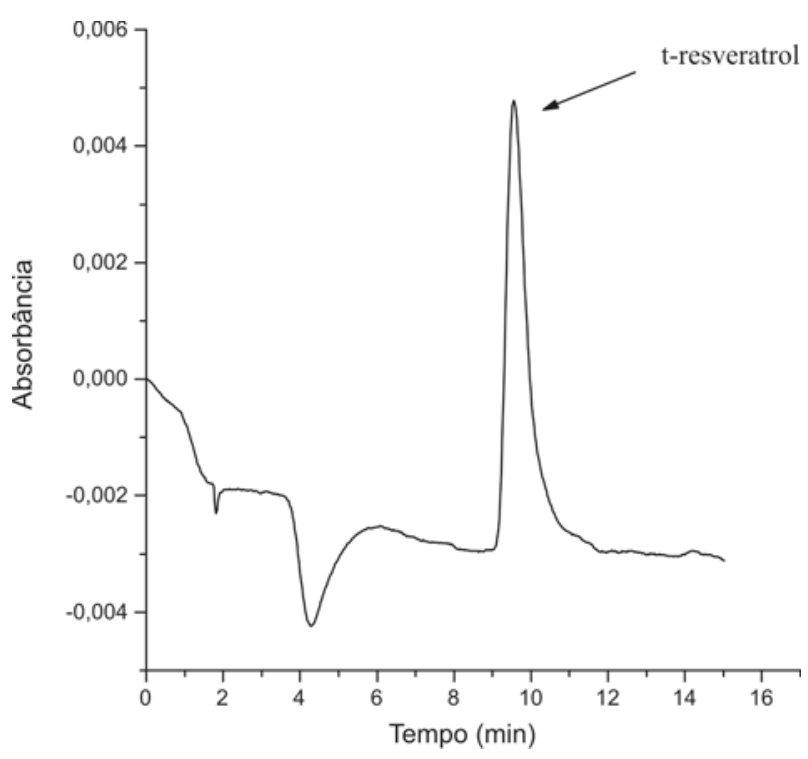

Figura 1. Cromatograma do trans-resveratrol em HPLC em suco de uva.

Tabela 2. Teores de resveratrol em sucos de uva obtidos das variedades produzidas nos sistemas orgânico e convencional expressos em $\mathrm{mg} \mathrm{L}^{-1}$

\begin{tabular}{lcc}
\hline Variedades & Orgânico & Convencional \\
\hline Concorde 766 & 25,9 & 32,5 \\
Rúbea 766 & 27,2 & 32,9 \\
\hline
\end{tabular}

Rev. Ceres, Viçosa, v. 57, n.1, p. 001-005, jan/fev, 2010 
A proporção encontrada nos sucos, como pode ser observada na Tabela 1, pode estar relacionada ao processo de cocção extrair maior número de substâncias da casca que outros métodos, como o do despolpamento. Segundo Cantos et al. (2001), é a porção da uva de incidência do resveratrol. Este autor descreve em seus experimentos a ausência dessa substância na polpa da uva.

$\mathrm{O}$ resveratrol é produzido pela videira em resposta às doenças causadas, por exemplo, ao ataque de Botrytis cinerea e Plasmospora viticola para inibir o progresso da infecção, o que o inclui numa classe de antibióticos chamada fitoalexina, também atua na planta como agente protetor contra raios ultravioletas e injúrias mecânicas (kolouchov-Hanzlikov et al., 2004). O teor de resveratrol presente na uva pode variar de acordo com as condições climáticas de cultivo, o ataque de fungos, cultivar, a safra e com o método de obtenção do suco (Nikfardjam et al., 2005); fato que pode justificar os menores teores encontrados para as variedades Concorde e Rúbea produzidas em sistema orgânico.

Segundo Jeandet et al. (1997), a produção de fitoalexinas é maior em cultivares que apresentam maior tolerância à doença do que naqueles mais suscetíveis. As variedades de uvas Concord e Rúbea, utilizadas neste experimento, são altamente vigorosas e resistentes a doenças (Maia \& Camargo, 2005), fator intrínseco da variedade que pode ter influenciado o alto teor de resveratrol presente no suco dessas uvas.

Romero-Perez et al. (2001) destacam que maior quantidade de resveratrol pode ser obtidas utilizando temperatura de $60^{\circ} \mathrm{C}$. Além da temperatura, as técnicas de vinificação e maceração da baga também interferem na concentração de resveratrol (Jeandet et al., 1995). É importante a realização de mais pesquisas em relação aos métodos de obtenção do suco, às técnicas de cultivo e às variedades que produzam concentrações significantes deste componente.

Atualmente tem-se dado grande ênfase às propriedades do resveratrol em relação à saúde humana, e sendo o suco de uva uma fonte desse elemento ele pode contribuir para uma alimentação saudável em contraste com o vinho que possui esse composto, mas também tem álcool.

As características químicas dos sucos de uvas mostram que as uvas estavam com acidez elevada, mas dentro do equilíbrio doce-ácido, o que está relacionado com o local de produção e os tratos culturais utilizados.

\section{CONCLUSÃO}

Por meio dos resultados obtidos pode-se concluir que os sucos de uva das variedades Concord e Rúbea apresentam elevado teor de resveratrol, independentemente do método de cultivo ser orgânico ou convencional. Porém em relação às outras características químicas do suco há necessidade de algumas melhorias para que o mesmo atenda a todas as exigências da legislação brasileira.

\section{AGRADECIMENTOS}

À CAPES, pelo apoio financeiro para a realização deste trabalho

\section{REFERÊNCIAS}

Brasil (1974) Ministério da Agricultura. Complementação dos padrões de identidade e qualidade para suco, refresco e refrigerante de: Uva. Publicada no D.O.U., Portaria n 371 de 19 de setembro de 1974. p.25-29

Cantos E, Espín JE \& Tomas-Barberán FA (2001) Postharvest induction modeling method using UV irradiation pulses for obtaining resveratrol-enriched table grapes: a new functional fruit? Journal of Agricultural and Food Chemistry, 49:50525058 .

Instituto de Tecnologia de Alimentos (1990) Análises químicas dos alimentos. Manual técnico. Campinas, Ital. 120p.

Jeandet P, Bessis R, Maume BF, Meunier P \& Trollat P (1995) Effect of enological practices on the resveratrol isomer content of wine. Journal of Agriculture and Food Chemistry, 43:316319.

Jeandet P, Breuil AC, Adrian M, Weston LA, Debord S, Meunier P, Maume G \& Bessis R (1997) HPLC Analysis of Grapevine Phytoalexins coupling photodiode array detection and fluorometry. Analytical Chemistry, 69:5172-5177.

Kolouchova-Hanzlikova I, Melzoch K, Filip V \& Smidrkal J (2004) Rapid method for resveratrol by HPLC with electrochemical and UV detections in wines. Food Chemistry, 87:151-158.

Lamuela-Raventós RM, Romero-Pérez AI, Waterhouse AL \& de la Torre-Bororat MC (1995) Direct HPLC analysis of cis- and trans-resveratrol and piceid isomers in Spanish red Vitis vinifera wines. Journal of Agriculture and Food Chemistry, 43:281- 283.

Maia JD \& Camargo UA (2005) Sistema de Produção de Uvas Rústicas para Processamento em Regiões Tropicais do Brasil. Embrapa Uva e Vinho, Sistema de Produção. Disponível em: $<$ http://sistemasdeproducao.cnptia.embrapa.br >. Acessado em: 20 de maio de 2007

Nikfardjam MSP, Laszló C \& Dietrich H (2006) Polyphenols, anthocyanins and trans-resveratrol in red wines from the Hungarian Villany region. Food Chemistry, 98:453-462.

Nikfardjam MSP, Laszló M, Avar P, Figler M \& Ohmacht R (2005) Resveratrol-derivatives and antioxidative capacity in wines made from botrytized grapes. Food Chemistry, 96:74-79.

Pezzi GM \& Fenocchio P (1976) Estudo analítico dos sucos de uva comerciais. Pesquisa Agropecuária Brasileira, 11:11-13.

Possier B, Cardova AC, Becquemin, JP \& Sumpio, BE (2005) Resveratrol inhibits vascular smooth muscle cell proliferation and induces apoptosis. Journal of Vascular Sugery, 42:9-13.

Rizzon LA (1998) Elaboração de suco de uva na propriedade vitícula. Bento Gonçalves, Embrapa Uva e Vinho. 24p. (Embrapa Uva e Vinho. Documentos, 21)

Rizzon LA \& Link M (2006) Composição do suco de uva caseiro de diferentes cultivares. Revista Ciência Rural, 36:689-692.

Rodriguez-Delgado MA, Gonzalez G, Perez-Trujillo JP \& GarcaMontelongo FJ (2002) Trans-resveratrol in wines from Canary Islands (Spain). Analysis by high performance liquid chromatography. Food Chemistry, 76:371-375.

Romero-Pérez AI, Lamuela-Raventós RM, Waterhouse AL \& de la Torre-Boronat MC (1996) Levels on cis- and trans-resveratrol and their glucosides in white and rosé Vitis vinifera wines from Spain. Journal of Agricultural and Food Chemistry, 44:2124-2128. 
Romero-Pérez AI, Ibern-Gómes M, Lamuelaraventós R.M \& Torre-Boronat MC (1999) Piceid, the major resveratrol derivative in grape juice. Journal of Agricultural and Food Chemistry, 47:1533-1536.

Romero-Perez AI, Lamuela-Raventós RM, André-Lacueva C \& De la Torre-Boronat MC (2001) Metod for quantitative extraction of reveratrol and piceid isomers in grape berry skins. Effect of powdery mildew on the stilbene content. Journal of Agricultural and Food Chemistry, 49:210-215.

Sautter CK, Denardin S, Alves A O, Mallmann CA, Penna NG \& Hecktheuer LH (2005) Determinação de resveratrol em sucos de uva no Brasil. Ciência e Tecnologia de Alimentos, 25:437-442.
Souto AH, Carneiro MC, Seferin M, Senna MJH, Conz A \& Gobbi K (2001) Determination of trans-resveratrol concentration in Brazilian red wines by HPLC. Journal of Food Composition and Analysis, 14:441-445.

Wang Y, Catana F, Yang Y, Roderick R \& Breemen RBV (2002) An Ln-MS Method for analyzing total resveratrol in grape juice, cranberry juice an wine. Journal of Agricultural and Food Chemistry, 50:431-435.

Yasui Y, Nakabayashi T, Naito A, Kawaguchi M, Yunoki K, Ohnishi M \& Ito S (1997) Changes in concentration of resveratrol during fermentation of musts from grapes grown in Tokachi. American Journal of Enology and Viticulture, 48:129. 